\title{
Loss of heterozygosity analysis of microsatellites on multiple chromosome regions in dysplasia and squamous cell carcinoma of the esophagus
}

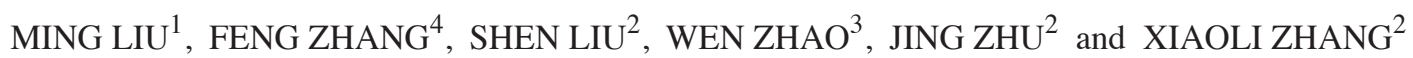 \\ Departments of ${ }^{1}$ Cardiothoracic Surgery, ${ }^{2}$ Clinical Laboratory Sciences, and ${ }^{3}$ Pathology, Affiliated South-West Hospital, \\ Third Military Medical University, Chongqing 400038; ${ }^{4}$ Beijing Institute of Genomics of the Chinese Academy \\ of Sciences, Beijing Genomics Institute, Beijing Proteomics Institute, Beijing 100029, P.R. China
}

Received May 10, 2011; Accepted June 16, 2011

DOI: 10.3892/etm.2011.297

\begin{abstract}
The objective of this study was to characterize the molecular events in the carcinogenesis of esophageal squamous cell carcinoma (ESCC) and to identify biomarkers for early detection of the disease. Matched precancerous and cancerous tissues resected from 34 esophageal cancer patients from Chongqing, southern China, were compared to evaluate the extent of loss of heterozygosity (LOH). Sixteen microsatellite markers on chromosome regions 3p, 4p, 5q, 8p, 9p, 9q, 11p, 13q and 17p were used for PCR-based LOH analysis. The overall frequency of $\mathrm{LOH}$ at the 16 microsatellite loci was significantly increased as the pathological status of the resection specimens changed from low-grade dysplasia (LGD) to high-grade dysplasia (HGD) and SCC $(\mathrm{P}<0.001)$. A total of 8 markers showed LOH in the LGD samples. In addition, heterozygosity was regained at 4 loci in the SCC samples of 4 patients, respectively, in comparison to the results for these loci in the HGD samples. The overall rate of $\mathrm{LOH}$ increased significantly with the deterioration of the lesions, indicating that tumorigenesis of the esophageal squamous epithelia is a progressive process involving accumulative changes in LOH. The 8 loci showing allelic loss in the LGD samples may be involved in the early-stage tumorigenesis of ESCC, and $\mathrm{LOH}$ analysis at these loci may help improve the early detection of this disease. Regain of heterozygosity found in certain patients suggests the possibility of genetic heterogeneity in the tumorigenesis of esophageal cancer.
\end{abstract}

\section{Introduction}

Esophageal cancer is a common type of malignant cancer in China. The 5-year survival rate of patients diagnosed with

Correspondence to: Dr Xiaoli Zhang, Department of Clinical Laboratory Sciences, Affiliated South-West Hospital, Third Military Medical University, Chongqing 400038, P.R. China

E-mail: xlzhang227@yahoo.com.cn

Key words: esophageal squamous cell carcinoma, microsatellite, loss of heterozygosity esophageal cancer is less than $15 \%$. Failure to make an early diagnosis is normally attributed to the absence of symptoms or the presence of mild symptoms during the early stages of the disease. Most of the patients are diagnosed in the intermediate or late stage of the disease. Although radical esophagectomy remains the primary treatment for esophageal cancer, it is quite difficult to treat patients with distant or lymph-node metastasis because of a tendency of postsurgical recurrence. Therefore, identification of biomarkers that improve the rates of early diagnosis of esophageal cancer or precursor lesions can significantly reduce mortality in esophageal cancer patients.

Similar to other solid tumors, chromosome loss is a common molecular defect in esophageal cancer. Chromosome regions with a high frequency of allelic loss indicate the presence of tumor-associated genes (such as tumor suppressors). A study of chromosome loss in dysplasia and early-stage esophageal cancer will aid in the discovery of major tumor-associated genes and will lead to the understanding of the tumorigenesis of esophageal cancer. They may also be used as potential biomarkers for early detection of esophageal cancer. Although extensive genomic instability has been found in patients with esophageal cancer, the molecular alterations that are closely related to the different stages of tumorigenesis have not been fully understood.

In order to further clarify the molecular alterations during the early-stage tumorigenesis of esophageal cancer, and to identify potential biomarkers for early detection of the disease, we analyzed allelic losses at a total of 16 microsatellite loci selected from chromosome regions 3p, 4p, 5q, 8p, 9p, 9q, 11p, $13 q$ and $17 p$ in matched squamous dysplasia and squamous cancer tissues resected from 34 esophageal cancer patients in Chongqing, southern China.

\section{Materials and methods}

Sample collection. Resection specimens from a total of 34 esophageal cancer patients were obtained from the Department of Thoracic Surgery, South-West Hospital, Chongqing, China. The patients included 23 men and 11 women with an average age of 58.6 years (range 44-74). All of the patients were newly diagnosed with esophageal cancer, 
A Blood DNA

\begin{tabular}{r|lll}
\hline$+Q$ & 120 & 140 & 160 \\
\hline 1200 & &
\end{tabular}

B

SCC DNA

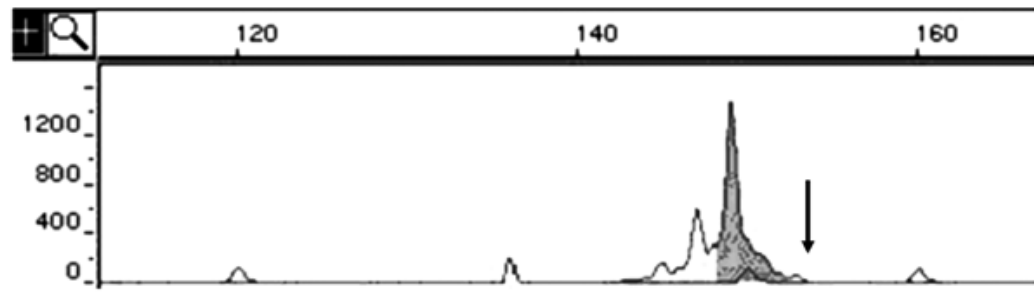

C

Blood DNA

\begin{tabular}{|c|c|c|}
\hline$+Q$ & 140 & 160 \\
\hline $1500_{-}$ & & \\
\hline 1200 _ & & \\
\hline 900 . & & \\
\hline 600. & & \\
\hline 300. & & \\
\hline
\end{tabular}

D

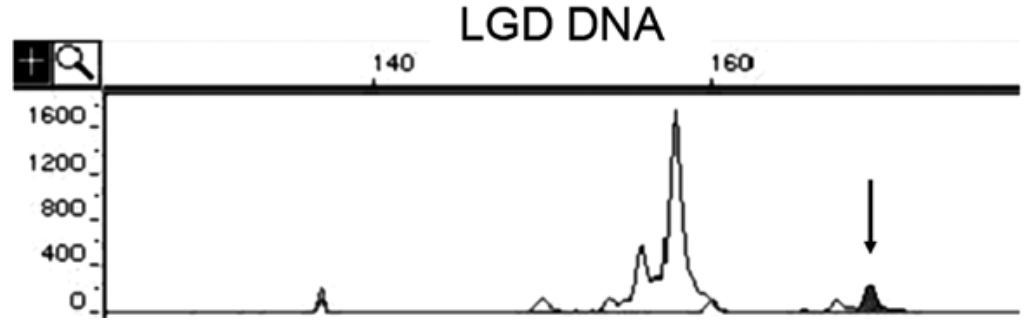

Figure 1. X-coordinate represents the size of the fragment and Y-coordinate represents the fluorescence intensity. (A and B) Allelotyping results of the D3S2452 locus from the peripheral blood samples and cancer tissue samples of a patient. DNA from the peripheral blood sample shows heterozygosity at the D3S2452 locus with fragments of two sizes, 149 and $153 \mathrm{bp}$, whereas DNA from the tumor tissue only shows a single fragment, 149 bp (arrowhead indicates the loss of a 153-bp fragment). These results indicate that LOH occurred at the D3S2452 locus in the cancer tissue. (C and D) Allelotyping results of the D4S174 locus from the peripheral blood and LGD-tissue samples of a patient. Both the peripheral blood and LGD samples comprise segments of two sizes, 157 and $169 \mathrm{bp}$, at the D4S174 locus; the fluorescence intensity at the 169-bp segment (arrowhead) in the LGD sample was reduced by at least 30\% of that in the peripheral blood sample. The results highlight the presence of LOH at the D4S174 locus in the LGD sample. SCC, squamous cell carcinoma; LGD, low-grade dysplasia, including mild and medium dysplasia (in this study).

had no previous history of any other malignant cancer and had not undergone previous radiotherapy or chemotherapy. The patients were Han Chinese who had lived in Chongqing for more than 20 years and did not have any direct kinship with each other. The resected samples were placed in liquid nitrogen immediately after the surgical removal and were preserved at $-80^{\circ} \mathrm{C}$ before use. In addition to the cancerous foci, surrounding tissues were also collected from the 34 patients. The tissue samples were pathologically diagnosed with mild, medium and severe dysplasia and squamous cell carcinoma (SCC) (1). This study classified mild and medium dysplasia as low-grade dysplasia (LGD), and severe dysplasia as high-grade dysplasia (HGD). In addition, peripheral blood samples were collected prior to surgery. Informed consent was obtained from all of the study subjects in accordance with the standards established by the local institutional review boards.

Tissue cell acquisition using microdissection. The frozen sections of squamous cancer tissue and dysplastic tissue $(5 \mu \mathrm{m})$ were stained with $\mathrm{H} \& \mathrm{E}$ and subjected to microdissection under a $x 40$ dissecting microscope to ensure that the proportion of tumor cells and dysplastic cells was $>80 \%$, which significantly reduced the rates of false positive or negative results during lthe oss of heterozygosity $(\mathrm{LOH})$ analysis.

Extraction of genomic DNA. Genomic DNA was extracted from the patient blood samples and tissues using the DNeasy Blood and Tissue kit (Qiagen). 
Table I. Frequencies of LOH at 16 microsatellite loci in the squamous dysplastic tissues and ESCC tissues.

\begin{tabular}{|c|c|c|c|c|}
\hline Marker & Location & LGD sample $(\%)^{\mathrm{a}}$ & HGD sample $(\%)^{\mathrm{a}}$ & SCC sample $(\%)^{\mathrm{a}}$ \\
\hline D3S1597 & $3 \mathrm{p} 25$ & 4/21 (19.0) & $12 / 22(54.5)$ & $18 / 22(81.8)$ \\
\hline D3S2452 & $3 \mathrm{p} 21-\mathrm{p} 14$ & $7 / 25(28.0)$ & $20 / 25(80.0)$ & $18 / 25(72.0)$ \\
\hline D3S1285 & $3 \mathrm{p} 14$ & $3 / 19(15.8)$ & 14/21 (66.7) & $16 / 21(76.2)$ \\
\hline D4S174 & 4p14-p13 & $2 / 27 \quad(7.4)$ & 7/27 (25.9) & $12 / 27(44.4)$ \\
\hline D5S409 & $5 q 14-q 15$ & $0 / 16 \quad(0.0)$ & $7 / 16(43.7)$ & $8 / 16(50.0)$ \\
\hline D5S2501 & $5 q 21-q 23.3$ & $1 / 13 \quad(7.7)$ & $7 / 13$ (53.8) & $7 / 13$ (53.8) \\
\hline D8S261 & 8p22-p21.3 & $0 / 18 \quad(0.0)$ & $5 / 18(27.8)$ & $6 / 18(33.3)$ \\
\hline D9S157 & 9p23-p22 & $0 / 23 \quad(0.0)$ & $16 / 23(69.5)$ & $15 / 23(65.2)$ \\
\hline D9S111 & 9q12-q21.1 & $0 / 25 \quad(0.0)$ & 9/25 (36.0) & $15 / 25(60.0)$ \\
\hline D9S125 & 9q34-q34 & 9/27 (33.3) & 21/27 (77.8) & 23/27 (85.2) \\
\hline D11S1338 & $11 \mathrm{p} 15.5$ & $0 / 29 \quad(0.0)$ & $10 / 29(34.5)$ & 11/29 (37.9) \\
\hline D13S175 & $13 q 13$ & $0 / 22 \quad(0.0)$ & $10 / 23(43.5)$ & $12 / 23(52.2)$ \\
\hline D13S153 & $13 q 14.2$ & 4/24 (16.7) & $10 / 24(41.7)$ & $13 / 24(54.2)$ \\
\hline D13S173 & 13q32-q34 & $0 / 24 \quad(0.0)$ & $5 / 24(20.8)$ & $11 / 24(45.8)$ \\
\hline D17S786 & 17 p13.1 & $5 / 23(21.7)$ & $13 / 23(56.5)$ & $16 / 23(69.5)$ \\
\hline D17S261 & $17 \mathrm{p} 11.2$ & $0 / 22 \quad(0.0)$ & $10 / 22(45.4)$ & $11 / 22(50.0)$ \\
\hline Total $^{\mathrm{b}}$ & & $35 / 358 \quad(9.8)$ & 176/362 (48.6) & $212 / 362(58.5)$ \\
\hline
\end{tabular}

${ }^{a}$ LGD, low-grade dysplasia, including mild and medium dysplasia (in this study); HGD, high-grade dysplasia, i.e., severe dysplasia; SCC, squamous cell carcinoma. ${ }^{\text {bIf }} \mathrm{P}<0.001$, then the difference is statistically significant.

Analysis of loss of heterozygosity. A total of 16 microsatellite loci were chosen from the 3p, 4p, 5q, 8p, 9p, 9q, 11p, 13q and 17p chromosome regions. The resected samples and paired blood samples from the 34 esophageal cancer patients were analyzed for $\mathrm{LOH}$ at the 16 loci. Information concerning these markers and primer sequences is available on the Genome Database (http://www.gdb.org/) and the NCBI genome database (http:// www.ncbi.nlm.nih.gov/). The primers were obtained as labeled primers with fluorescent dye at the 5-terminus (MWG Biotech, Ebersberg, Germany).

$\mathrm{LOH}$ analysis was performed as described in our previous study (2). In brief, the 5- $\mu 1$ volume of microsatellite DNA amplification included $40 \mathrm{ng}$ of DNA template, 1X Buffer, $200 \mu \mathrm{M}$ dNTPs, $250 \mathrm{nM}$ microsatellite primer, $2.5 \mathrm{mM}$ $\mathrm{MgCl}_{2}$ and 0.25 units of Taq DNA polymerase. The PCR conditions were as follows: pre-denaturation at $94^{\circ} \mathrm{C}$ for $3 \mathrm{~min}$, followed by 30 cycles of $94^{\circ} \mathrm{C}$ for $30 \mathrm{sec}, 55-60^{\circ} \mathrm{C}$ for $30 \mathrm{sec}$, $72^{\circ} \mathrm{C}$ for $1 \mathrm{~min}$ and a final elongation step at $72^{\circ} \mathrm{C}$ for $4 \mathrm{~min}$. PCR products were loaded on a fluorescent sequencer gel (ABI PRISM 377). The images were captured, converted and analyzed using GeneScan software, and the size of each allele fragment was automatically calculated.

When the peripheral blood DNA of a patient showed heterozygosity at a certain locus, the locus was marked as informative. The tissue sample of the patient was considered to show LOH when the DNA from the sample showed homozygosity at the informative locus or when the fluorescence intensity of one of the two alleles was $<30 \%$ of that in the blood DNA of the patient (Fig. 1). The frequency of $\mathrm{LOH}$ at each locus was defined as the total number of tissue samples with $\mathrm{LOH}$ at this locus/the total number of informative samples.
Statistical analysis. The LOH frequencies at each locus between the different groups were compared using the $\chi^{2}$ test, and a P-value $<0.05$ was considered statistically significant.

\section{Results}

The results of the present study are shown in Table I. The overall frequencies of LOH at the 16 microsatellite loci significantly increased as the pathological status of the resection samples changed (from LGD to HGD and to SCC). The overall frequency of LOH in the LGD samples (9.8\%) was significantly lower than that in the HGD (48.6\%) and SCC (58.5\%) samples $(\mathrm{P}<0.001)$. Although the $\mathrm{LOH}$ frequency at each locus showed an increasing trend with increasing disease severity, the difference was not statistically significant. Eight loci (D3S1597, D3S2452, D3S1285, D4S174, D5S2501, D9S125, D13S153 and D17S786) showed LOH in the informative LGD samples, and another 8 loci were found to present $\mathrm{LOH}$ in the informative HGD specimens. All 16 loci were found to have $\mathrm{LOH}$ in the SCC samples. Furthermore, by comparing the occurrence of $\mathrm{LOH}$ in samples with different pathological statuses from the same patient, we found regain of heterozygosity at loci D3S2452, D4S174, D9S125 and D17S261, in the SCC samples of 4 patients, respectively, compared to the HGD specimens which showed $\mathrm{LOH}$ at the corresponding loci.

\section{Discussion}

A total of 16 highly polymorphic microsatellite markers from 9 chromosome regions with a high frequency of allelic loss in esophageal cancer were selected. Surgically resected squamous dysplasia and SCC samples from 34 esophageal 
cancer patients were subjected to $\mathrm{LOH}$ analysis at these loci. The results showed that the overall frequencies of $\mathrm{LOH}$ at the 16 microsatellite loci significantly increased as the pathological status of the resection specimens deteriorated (i.e., from LGD to HGD and to SCC). These results indicated that tumorigenesis of esophageal squamous epithelia is a progressive process involving a series of molecular alterations. As the alterations accumulate to a certain degree, the cell morphology and behavior undergo a radical change, leading to malignancy (3). This finding was consistent with previous studies involving other tumor types, such as prostate, colon and breast cancer (4-7). Moreover, the results were also in accordance with those of a previous study showing that increasing grades of dysplasia were associated with an increased risk of developing ESCC (8).

Of the 16 loci, 8 loci were found to show LOH in LGD, indicating that $\mathrm{LOH}$ at these 8 loci may be involved in the early-stage tumorigenesis of ESCC, and that these specific loci may be used as markers for the screening of esophageal cancer. At present, certain tumor-associated genes have been identified near these loci, such as FHIT (3p), RASSF1A (3p), APC (5q), ANXI (9q), DECl (9q), RBI (13q), BRCA2 (13q), ING1 (13q) and TP53 (17p). The expression of ANXI in chromosome $9 \mathrm{q}$ has been reported to be closely related to tumor progression in patients with breast cancer and possibly plays an important role in the early stage of tumorigenesis (9). Study concerning this gene in esophageal cancer showed that its expression at the protein level in cancer tissues was significantly lower than that in normal tissues (10). Another candidate gene, $D E C 1$ at chromosome $9 \mathrm{q}$, has been reported to be down-regulated in esophageal cancer, and transfection of the cDNA of DECl may inhibit the proliferation of various types of cancer cells, suggesting that it may participate in the development of esophageal cancer (11). The chromosome $13 q$ region has a high frequency of allelic loss in esophageal cancer. However, the identified candidate genes within this region, such as $R B 1, B R C A 2$ and $I N G 1$, seldom show mutations in esophageal cancer, implying that other unknown tumor-suppressor genes within this region may participate in the tumorigenesis of this cancer (12). The chromosome $17 \mathrm{p}$ region involving the $P 53$ locus also shows a high frequency of allelic loss in esophageal cancer. Studies of the P53 gene in esophageal cancer have shown that $\mathrm{LOH}$ and mutations are the leading causes of its inactivation, which is in accordance with the 'two-hit' model of tumorigenesis indicating that P53 is a major tumor suppressor in esophageal cancer (13).

In addition, 8 other loci were found to show $\mathrm{LOH}$ in the HGD samples, indicating that $\mathrm{LOH}$ at these loci may be involved in the late stages of tumorigenesis (such as invasion and metastasis) in esophageal cancer.

This study also compared the occurrence of $\mathrm{LOH}$ in samples of different pathological statuses from the same patient. Notably, in 4 patients $\mathrm{LOH}$ was found at certain loci in the HGD samples, whereas heterozygosity was regained at the same loci in matched SCC samples. The regain of heterozygosity at some loci in tumor tissues indicated that the tumorigenesis of esophageal cancer may show genetic heterogeneity, i.e., the HGD and SCC samples of the same patient may have been derived from different tumor-cell clones. Cells with different molecular defects may have distinct tumorigenesis processes, leading to different tumor-cell clones. A similar result has been found in adenocarcinoma of the esophagus; the molecular defects found in the severe dysplastic lesions were not detected in the invasive adenocarcinoma lesions of the same patient (14). A study involving patients with oral squamous cancer has also shown that most of the molecular alterations found in the precursor lesions were not revealed in the matched tumor tissues (15). Similar observations have been reported in studies on other cancers, such as breast and prostate cancer $(16,17)$.

Finally, it is worth mentioning that in several LGD samples from our subjects, there were already multiple loci exhibiting allelic loss, indicating that the earliest molecular event in esophageal cancer may occur in histologically normal squamous epithelia. In a recent study on esophageal cancer, $19 \%$ of the histologically normal epithelia within the abnormal mucosal region (unstained with iodine) already showed $\mathrm{LOH}$ alterations, suggesting that the histologically normal epithelia adjacent to the tumor tissues may have early-stage $\mathrm{LOH}$ alterations, which may be necessary for the tumorigenesis of esophageal cancer (18). Although, at present, histological examination remains an effective method for the early detection of cancer, molecular tests, such as $\mathrm{LOH}$ analysis of certain specific loci at the early stage, can be a promising and rational strategy for the early diagnosis or prediction of esophageal cancer.

\section{Acknowledgements}

This study was supported, in part, by a grant from the Chongqing Local Natural Sciences Foundation of China (2010BB5192).

\section{References}

1. Dawsey SM,Lewin KJ,Liu FS, Wang GQ and Shen Q: Esophageal morphology from Linxian, China. Squamous histologic findings in 754 patients. Cancer 73: 2027-2037, 1994.

2. Zhang XL, Fu WL, Zhao HX, Zhou LX, Huang JF and Wang JH: Molecular studies of loss of heterozygosity in Chinese sporadic retinoblastoma patients. Clin Chim Acta 358: 75-80, 2005.

3. Maley CC, Galipeau PC, Li X, et al: The combination of genetic instability and clonal expansion predicts progression to esophageal adenocarcinoma. Cancer Res 64: 7629-7633, 2004.

4. Maitra A, Wistuba II, Washington C, et al: High-resolution chromosome $3 \mathrm{p}$ allelotyping of breast carcinomas and precursor lesions demonstrates frequent loss of heterozygosity and a discontinuous pattern of allele loss. Am J Pathol 159: 119-130, 2001.

5. Wang Z and Lai FM: [Analysis of loss of heterozygosity on chromosome 8 in human prostate carcinoma and high grade prostatic intraepithelial neoplasia]. Zhonghua Nan Ke Xue 10: 26-28; 31 , 2004.

6. Karakosta A, Golias C, Charalabopoulos A, Peschos D, Batistatou A and Charalabopoulos K: Genetic models of human cancer as a multistep process. Paradigm models of colorectal cancer, breast cancer, and chronic myelogenous and acute lymphoblastic leukaemia. J Exp Clin Cancer Res 24: 505-514, 2005.

7. Ha PK, Pilkington TA, Westra WH, Sciubba J, Sidransky D and Califano JA: Progression of microsatellite instability from premalignant lesions to tumors of the head and neck. Int $J$ Cancer 102: 615-617, 2002.

8. Dawsey SM, Lewin KJ, Wang GQ, et al: Squamous esophageal histology and subsequent risk of squamous cell carcinoma of the esophagus. A prospective follow-up study from Linxian, China. Cancer 74: 1686-1692, 1994.

9. Shen D, Nooraie F, Elshimali Y, et al: Decreased expression of annexin A1 is correlated with breast cancer development and progression as determined by a tissue microarray analysis. Hum Pathol 37: 1583-1591, 2006. 
10. Zhou G,Li H, DeCamp D, et al: 2D differential in-gel electrophoresis for the identification of esophageal scans cell cancer-specific protein markers. Mol Cell Proteomics 1: 117-124, 2002.

11. Yang L, Leung AC, Ko JM, et al: Tumor suppressive role of a $2.4 \mathrm{Mb} 9 \mathrm{q} 33-\mathrm{q} 34$ critical region and DEC1 in esophageal squamous cell carcinoma. Oncogene 24: 697-705, 2005

12. Huang XP, Wei F, Liu XY, et al: Allelic loss on 13q in esophageal squamous cell carcinomas from northern China. Cancer Lett 185: 87-94, 2002.

13. Hu N, Huang J, Emmert-Buck MR, et al: Frequent inactivation of the TP53 gene in esophageal squamous cell carcinoma from a high-risk population in China. Clin Cancer Res 7: 883-891, 2001.

14. Koppert LB, Wijnhoven BP, van Dekken H, Tilanus HW and Dinjens WN: The molecular biology of esophageal adenocarcinoma. J Surg Oncol 92: 169-190, 2005.
15. Partridge M, Pateromichelakis S, Phillips E, Emilion G and Langdon J: Profiling clonality and progression in multiple premalignant and malignant oral lesions identifies a subgroup of cases with a distinct presentation of squamous cell carcinoma. Clin Cancer Res 7: 1860-1866, 2001.

16. Cheng L, Bostwick DG, Li G, et al: Allelic imbalance in the clonal evolution of prostate carcinoma. Cancer 85: 2017-2022, 1999.

17. Heim S, Teixeira MR, Dietrich CU and Pandis N: Cytogenetic polyclonality in tumors of the breast. Cancer Genet Cytogenet 95: 16-19, 1997.

18. He S, Guo GM, Liu FX, et al: Molecular analysis in combination with iodine staining may contribute to the risk prediction of esophageal squamous cell carcinoma. J Cancer Res Clin Oncol 134: 307-315, 2008. 